\title{
Prolactin levels are positively correlated with polychlorinated biphenyls (PCB) in cord serum
}

\author{
L. Takser ${ }^{1}$, J. Lafond ${ }^{2} \&$ D. Mergler ${ }^{3}$ \\ ${ }^{I}$ Departement Obstétrique et Gynecologie, Université de Sherbrooke, \\ Sherbrooke, Quebec, Canada \\ ${ }^{2}$ Laboratoire de Physiologie Materno-Fotale, \\ Université du Québec à Montréal, Montréal, Quebec, Canada \\ ${ }^{3}$ CINBIOSE, Université du Québec à Montréal, \\ Montréal, Quebec, Canada
}

\begin{abstract}
Polychlorinated biphenyl compounds (PCB) are global environmental contaminants that cause the disruption of endocrine and nervous systems in animals and humans. However, little evidence exists showing their potential interference with nervous system development following in utero exposure to low PCB levels. The aim of this study is to examine cord serum prolactin (PRL) levels in relation to cord blood concentrations of 14 PCB congeners in healthy women recruited during pregnancy. Our results showed a significant increase of cord serum PRL with an increasing level of PCB-153, the most prevalent PCB congener. Thus, knowing that PRL release is under inhibitory dopamine regulation, our results suggest that low level exposure to a mixture of persistent environmental contaminants could interfere with dopaminergic transmission in the fetal brain.
\end{abstract}

Keywords: $P C B$, prolactin, cord blood, environment.

\section{Introduction}

PCB are global pollutants characterized by their resistance to metabolic degradation, their persistence contributes to their accumulation at the top of food chain. Potential mechanisms underlying PCB-induced developmental neurotoxicity are still unclear, but significant effects on dopamine metabolism and thyroid function have been reported $[1,2]$. In vitro studies suggest that signal transduction pathways can be disrupted by PCB [3]. Recently, an interaction of 
PCB with dopamine transporters and vesicular monoamine transporters, as well as oxidative mechanisms of dopaminergic toxicity, have been reported $[4,5]$. Unlike to the non coplanar congeners, the coplanar and mono-ortho-substituted PCB have been shown to alter the signaling pathway via an aryl hydrocarbon receptor (AhR) mechanism [6].

Although it exists an increasing evidence of PCB effects on neurodevelopment, there is no sufficient data about congener-specific PCB neurotoxic effects in human [7]. The objective of our exploratory study was to examine the relationship between low level of environmental exposure to PCB and cord serum PRL level, which is used as an indirect marker of dopaminergic transmission. In humans, fetal PRL secretion begins early (7.5-10 weeks) during fetal development, is independent of maternal PRL levels and is dopamine dependent [8]. Considering the structure-specific mechanisms of PCB toxicity, we analysed and evaluated the relationship between PRL and PCB structure, distinguishing levels of coplanar (mono-ortho-congeners) or non-coplanar PCB congeners separately.

\section{Materials and methods}

\subsection{Studied population}

The women participating in this study were recruited during their first prenatal visit at the Centre for Local Community Services (CLSC, part of the National Public Health System) in the Southwestern region of Québec. The study was approved by Ethical Committee of Université du Québec à Montréal. Inclusion criteria for recruitment were no history of workplace exposure to toxic chemicals and no history of previous chronic illness, such as diabetes and/or other endocrine pathologies, arterial hypertension, or cancer. After signing a consent form, an interview administered questionnaire, which contained general sociodemographic data and information on residency, medical history, drinking and smoking habits and diet, was filled out and blood samples were obtained.

From 310 pregnant women invited to participate, $246(79 \%)$ have accepted to participate, 42 were excluded according exclusion criteria and 47 were also excluded because their advanced gestational age. Finally, from 157 pregnant women recruited in two CLCS, 6 had spontaneous abortions and 101 gave birth in the hospital selected for the final step of the study. Two weeks following birth, a $2^{\text {nd }}$ questionnaire was interview administered to all women who delivered at the selected hospital or not. This second questionnaire included information on medical and obstetrical history, birth data, as well as smoking and drinking habits during pregnancy. The characteristics of women who had not delivered in the selected hospital were not significantly different.

\subsection{Biological sampling}

Maternal blood and cord blood samples were collected at the hospital at delivery. Serum samples were kept at $-20^{\circ} \mathrm{C}$ until contaminant or hormone assays (within 4 months maximum). 


\subsection{PCB determination}

Laboratory analyses were performed by the Centre de Toxicologie du Québec (CTQ) by a GC-MS method, using Gas Chromatograph 6890 and mass spectroscopy detector 5973 from Agilent (Mississauga, Ontario). Two $\mathrm{ml}$ of blood (maternal or cord) were extracted using an ammonium sulfate/ethanol/hexane mixture, cleaned-up on Florisil columns and taken to a final volume of $100 \mu \mathrm{L}$. Routine checks of accuracy and precision were accomplished using reference materials from National Institute of Standards and Technology. Also, periodic evaluations were accomplished by the participation of CTQ to two external proficiency testing programs. The detection limit for all PCB congeners, according the chosen method, was $0.02 \mu \mathrm{g} / \mathrm{L}$.

\subsection{Lipid determination}

Total cholesterol (TC), free cholesterol (FC), triglyceride (TG) and phospholipid (PL) levels were determined in serum by enzymatic methods (Randox CH 202, Crumlin, UK) using the Technicon RA-500 analyzer (Bayer Corporation Inc, Tarrytown, NY). Serum total lipids were calculated using the summation method: total lipids $=1.677(\mathrm{TC}-\mathrm{FC})+\mathrm{FC}+\mathrm{TG}+\mathrm{PL}[9]$.

\subsection{PRL determination}

Cord serum PRL levels were determined by an automatic enzymo-immunologic assay using Elecsys automate (Roche Diagnostics). The maximum limit level for PRL was $500 \mathrm{ng} / \mathrm{mL}$.

\subsection{Statistical analyses}

All statistical analyses were performed using SAS version 8.12 [10]. Only 86 subjects have complete data (PCB, PRL and questionnaire), and all presented results were obtained on these 86 subjects. In order to include subjects with nondetected values in statistical analysis, the half of the detection limit was attributed to these subjects. Relationships between PCB and PRL were tested using ANCOVA (GLM procedure) and correlation analysis (CORR procedure) taking into account maternal tobacco smoking during pregnancy and month of delivery (because known PRL seasonal variation [11]). Total lipids concentrations were introduced to all models as fixed model parameters. The normality of distribution of residuals was checked for all models. The relationships between PCB and PRL were presented for adjusted for lipids models and those without adjustment.

Previously, we reported for the same population, a relationship between cord blood manganese and PRL [12]. Thus, manganese exposure variable was introduced in all models, but it did not modify our results (data not shown).

The following PCB exposure variables were used in analysis: - total PCB (sum of all congeners), - mono-ortho-coplanars (sum of CB-105, CB-118, and 
CB-156) and - non-coplanar PCB congeners (CB-138, CB-153, CB-180) individually. CB-101 and CB-28 were excluded from statistical analysis because $100 \%$ were undetected values.

\section{Results}

\subsection{Population characteristics and PCB levels}

The maternal average age was 26 years old (range 17 - 39); 23 women $(27 \%)$ smoked during pregnancy and nine $(10 \%)$ consumed alcohol moderately $(0.5-2$ drink/week, 4-30g alcohol/week). During pregnancy, ten women $(10 \%)$ had gestational diabetes, among them 2 had pregnancy induced hypertension; 10 women had pregnancy induced hypertension without gestational diabetes, and among these ten, two had proteinuria. Eight (9\%) women delivered by cesarean, peridural anesthesia was performed in 65 cases and sedative therapy was used in 36 deliveries. Four deliveries (4.6\%) occurred before 37 weeks of pregnancy, the average birth weight was $3.3 \mathrm{~kg}$ (range $2.1-5.0 \mathrm{~kg}$ ) and $51 \%$ of newborns were boys.

The PCB data are given in Table 1. Total PCB in cord blood is highly correlated with maternal serum PCB (Spearman $r=0.74$ and $0.73, p<0.0001$ for PCB values uncorrected and corrected with total lipids, respectively). Maternal PCB values uncorrected for total lipids were significantly higher than those from cord serum (paired $\mathrm{t}=11.4, \mathrm{p}<0.0001$ ). However, this relation was inverse for PCB values corrected with total lipids (paired $\mathrm{t}=-10.6, \mathrm{p}<0.0001$ ).

Table 1: $\quad$ Cord serum PCB concentrations, $\mu \mathrm{g} / \mathrm{L}$.

\begin{tabular}{|c|c|c|c|}
\hline Congeners & Median & $5^{\text {th }}-95^{\text {th }}$ percentiles & Maximum value \\
\hline$\Sigma$ PCB & 0.16 & nd-0.35 & 0.38 \\
\hline $\begin{array}{c}\sum \text { mono-ortho- } \\
\text { coplanars } \\
(\mathrm{CB}-105, \mathrm{CB}-118, \mathrm{CB}-156)\end{array}$ & 0.04 & nd-0.07 & 0.13 \\
\hline $\mathrm{CB}-138$ & 0.02 & $\mathrm{nd} *-0.06$ & 0.07 \\
\hline CB-153 & 0.02 & $\mathrm{nd}-0.08$ & 0.10 \\
\hline CB-180 & $\mathrm{nd}$ & $\mathrm{nd}-0.05$ & 0.06 \\
\hline
\end{tabular}

*undetected value.

The median value of cord serum PRL was $372.3 \mathrm{ng} / \mathrm{mL}$, ranging from $146 \mathrm{ng} / \mathrm{mL}$ to the maximal possible level $(500 \mathrm{ng} / \mathrm{mL})$ according the assay 
conditions; 25 samples were equal or above this level. Cord serum PRL levels were not significantly related to mode of delivery, pregnancy pathologies or medication.

\subsection{Relation of cord blood PCB to PRL}

The relationships between cord blood PCB exposure and PRL levels are presented in Table 2. Sum of PCB is positively correlated to cord serum PRL levels (partial Pearson $\mathrm{r}=0.22, \mathrm{p}<0.05$ ). Mono-ortho-chlorinated PCB congeners are not related to PRL levels. Among non-coplanar congeners, only CB-153 reaches the significance level (partial Pearson $r=0.23$, $p<0.05$ ).

Table 2: PRL levels in relation to PCB concentrations in cord blood (General Linear Model estimates).

\begin{tabular}{|c|c|c|}
\hline \multirow{2}{*}{ Cord blood PCB } & \multicolumn{2}{|c|}{ PRL, ng/mL } \\
\hline & $\begin{array}{l}\text { Unadjusted for lipids } \\
\text { models }\end{array}$ & $\begin{array}{l}\text { Adjusted for lipids } \\
\text { models* }\end{array}$ \\
\hline $\begin{array}{ll}\Sigma P C B & \\
& \beta \\
& p \\
\end{array}$ & $\begin{array}{c}492.9 \\
0.04\end{array}$ & $\begin{array}{c}379.5 \\
0.07\end{array}$ \\
\hline $\begin{array}{c}\text { Emono-ortho-coplanars } \\
\beta \\
\mathrm{p} \\
\end{array}$ & $\begin{array}{c}1251.4 \\
0.10 \\
\end{array}$ & $\begin{array}{c}1155.9 \\
0.12 \\
\end{array}$ \\
\hline CB-138 & $\begin{array}{c}1139.3 \\
0.12 \\
\end{array}$ & $\begin{array}{c}1014.6 \\
0.17 \\
\end{array}$ \\
\hline CB-153 & $\begin{array}{c}1284.0 \\
0.03 \\
\end{array}$ & $\begin{array}{c}1191.9 \\
0.04 \\
\end{array}$ \\
\hline CB-180 & $\begin{array}{c}1270.2 \\
0.25\end{array}$ & $\begin{array}{c}1102.4 \\
0.32\end{array}$ \\
\hline
\end{tabular}

* The lipid concentrations were introduced in linear models as fixed variable. All models were adjusted for tobacco smoking during pregnancy and month of birth (degree of freedom was 3;80 for unadjusted for lipids models and 4;78 for adjusted for lipids models).

\section{Discussion}

Our study is the first to show a relationship between PRL levels in cord serum and PCB exposure in utero. The finding that cord serum PRL levels increase in relation to exposure to environmental PCB suggests that these contaminants can interfere with fetal dopamine metabolism. Dopamine is the primary 
neuroendocrine factor that regulates the PRL secretion. Thus, removal of endogenous dopaminergic tone by catecholamine depletion or blockade of the dopamine $\left(\mathrm{D}_{2}\right)$ receptors on lactotrophs results in a significant elevation of circulating PRL levels [13]. Although many other peptides, such as thyrotropinreleasing hormone (TRH), vasoactive intestinal peptide (VIP), oxytocin or endothelin [14], are known to regulate PRL secretion, their actions are not primary responsible for the increase of PRL secretion. Thus, TRH stimulation mechanism could be hypothesized, but in our population, no significant relation was observed between PCB and thyroid-stimulating hormone (TSH) levels (regulated by TRH) (data not shown). Considering the available literature, the only explanation for our finding remains the dopaminergic mechanism.

According Richardson and Miller [5], the observed relationship between PRL and PCB was more significant in relation to $\mathrm{CB}-153$, a non coplanar congener, than to mono-ortho-chlorinated congeners, which do not interact with dopaminergic transmission. Moreover, in vitro [1] and in vivo studies on dopaminergic toxicity induced by PCB, especially with non coplanar congeners, were reported in adult $[15,16]$, in developing animal and in low doses prenatal exposure (without systemic effects) [17]. However, the sum of PCB was more significantly related to PRL, than individual congeners, suggesting the additive effect of individual congeners in PCB mixture. In addition, high PCB concentrations were found in caudate nuclei from post mortem Parkinson disease patients, suggesting their causal role in dopamine loss in humans [18].

Few experimental data are available about chronic in utero exposure to $\mathrm{PCB}$ and their effects on dopamine or PRL secretion. Lyche et al. (2004) observed no significant effect on PRL levels of female goat prenatally exposed to low doses of CB-126 and CB-153 (similar to environmental doses) [19]. However, acute exposure to non persistent non coplanar congeners leads to transitory rise of blood PRL levels and to decrease of hypothalamic dopamine levels in developing rats [20]. In adult man, high dietary exposure to PCB and pesticides, from fish consumption, was not linked to circulating levels of PRL [21]. All this information does not exclude our suggestion that PCB exposure in utero can be related to the increase of PRL level in human fetuses. In fact, inter- and intraspecies differences should be considered regarding the neurotoxic susceptibility in relation to PCB exposures, as well as to the differences in prenatal development (singleton pregnancy in primates, length of gestation, i.e. exposure duration, type of placentation).

Our study presents some limitations. Firstly, for 25 samples, the PRL concentration was above the maximum limit of detection, of our PRL assay and a value of $500 \mathrm{ng} / \mathrm{mL}$ was assigned to these samples. The literature data report many different PRL values in cord blood, but Parker et al. (1986) reported an average cord serum PRL concentration in full term newborns that is comparable to our results. But it is important to note that some authors also reported lower levels [2]. Secondly, retrospectively calculated statistical power of study sample was not sufficient: $56 \%$ calculated for alpha error 0.05 and observed correlation of 0.23 .

In addition, exposure levels to PCB congeners in our population were 3-45 times lower than in previous reported studies reviewed by Longnecker et al. [22]. 
And, only CB-153, the most prevalent congener, as well as the sum of PCB congeners, were significantly related to PRL. The lack of statistical association with other PCB congeners can be related to very low levels of exposure or to lack of statistical power. Thus, there is a need for further investigations using newborns from more exposed populations.

The present study, despite limitations, is of major interest given limited scientific literature available about biomarkers of neurotoxicity in human fetuses. Knowing that developmental exposures to dopaminergic disruptors can result in long term behavioral modifications, it is important to develop strategies to determine in vivo biomarkers of dopaminergic toxicity induced by pollutants present in general population environment.

\section{References}

[1] Shain, W., Bush, B. \& Seegal, R., Neurotoxicity of polychlorinated biphenyls: structure-activity relationship of individual congeners. Toxicol Appl Pharmacol, 111, pp. 33-42, 1991.

[2] Seegal, R.F., The neurotoxicological consequences of developmental exposure to PCBs. Toxicol Sci, 57, pp. 1-3, 2000.

[3] Inglefield, J.R., Mundy, W.R. \& Shafer, T.J., Inositol 1,4,5-triphosphate receptor-sensitive $\mathrm{Ca}(2+)$ release, store-operated $\mathrm{Ca}(2+)$ entry, and cAMP responsive element binding protein phosphorylation in developing cortical cells following exposure to polychlorinated biphenyls. J Pharmacol Exp Ther, 297, pp. 762-773, 2001.

[4] Lee, D.W. \& Opanashuk, L.A., Polychlorinated biphenyl mixture aroclor 1254-induced oxidative stress plays a role in dopaminergic cell injury. Neurotoxicology, 25, pp. 925-939, 2004.

[5] Richardson, J.R. \& Miller, G.W., Acute exposure to aroclor 1016 or 1260 differentially affects dopamine transporter and vesicular monoamine transporter 2 levels. Toxicol Lett, 148, pp. 29-40, 2004.

[6] Van den Berg, M., Birnbaum, L., Bosveld, A.T., Brunstrom, B., Cook, P., Feeley, M., Giesy, J.P., Hanberg, A., Hasegawa, R., Kennedy, S.W., Kubiak, T., Larsen, J.C., van Leeuwen, F.X., Liem, A.K., Nolt, C., Peterson, R.E., Poellinger, L., Safe, S., Schrenk, D., Tillitt, D., Tysklind, M., Younes, M., Waern, F. \& Zacharewski, T., Toxic equivalency factors (TEFs) for PCBs, PCDDs, PCDFs for humans and wildlife. Environ Health Perspect, 106, pp. 775-792, 1998.

[7] Schantz, S.L., Widholm, J.J. \& Rice, D.C., Effects of PCB exposure on neuropsychological function in children. Environ Health Perspect, 111, pp. 357-576, 2003.

[8] Freemark, M., Ontogenesis of prolactin receptors in the human fetus: roles in fetal development. Biochem Soc Trans, 29, pp. 38-41, 2001.

[9] Patterson, D.G., Jr., Isaacs, S.G., Alexander, L.R., Turner, W.E., Hampton, L., Bernert, J.T. \& Needham, L.L., Determination of specific polychlorinated dibenzo-p-dioxins and dibenzofurans in blood and 
adipose tissue by isotope dilution-high-resolution mass spectrometry. IARC Sci Publ, 299-342, 1991.

[10] SAS Institute Inc., SAS/STAT User's Guide, version 8. 1999: Cary, NC. 3884.

[11] Houghton, D.C., Young, I.R. \& McMillen, I.C., Photoperiodic history and hypothalamic control of prolactin secretion before birth. Endocrinology, 138, pp. 1506-1511, 1997.

[12] Takser, L., Mergler, D., de Grosbois, S., Smargiassi, A. \& Lafond, J., Blood manganese content at birth and cord serum prolactin levels. Neurotoxicol Teratol, 26, pp. 811-815, 2004.

[13] Lamberts, S.W. \& Macleod, R.M., Regulation of prolactin secretion at the level of the lactotroph. Physiol Rev, 70, pp. 279-318, 1990.

[14] Samson, W.K., Taylor, M.M. \& Baker, J.R., Prolactin-releasing peptides. Regul Pept, 114, pp. 1-5, 2003.

[15] Seegal, R.F., Bush, B. \& Brosch, K.O., Comparison of effects of Aroclors 1016 and 1260 on non-human primate catecholamine function. Toxicology, 66, pp. 145-163, 1991.

[16] Seegal, R.F., Bush, B. \& Brosch, K.O., Sub-chronic exposure of the adult rat to Aroclor 1254 yields regionally-specific changes in central dopaminergic function. Neurotoxicology, 12, pp. 55-65, 1991.

[17] Seegal, R.F., Brosch, K.O. \& Okoniewski, R.J., Effects of in utero and lactational exposure of the laboratory rat to $2,4,2^{\prime}, 4^{\prime}-$ and $3,4,3^{\prime}, 4^{\prime}-$ tetrachlorobiphenyl on dopamine function. Toxicol Appl Pharmacol, 146, pp. 95-103, 1997.

[18] Corrigan, F.M., Murray, L., Wyatt, C.L. \& Shore, R.F., Diorthosubstituted polychlorinated biphenyls in caudate nucleus in Parkinson's disease. Exp Neurol, 150, pp. 339-342, 1998.

[19] Lyche, J.L., Skaare, J.U., Larsen, H.J. \& Ropstad, E., Levels of PCB 126 and PCB 153 in plasma and tissues in goats exposed during gestation and lactation. Chemosphere, 55, pp. 621-629, 2004.

[20] Khan, M.A., Lichtensteiger, C.A., Faroon, O., Mumtaz, M., Schaeffer, D.J. \& Hansen, L.G., The hypothalamo-pituitary-thyroid (HPT) axis: a target of nonpersistent ortho-substituted PCB congeners. Toxicol Sci, 65, pp. 52-61, 2002.

[21] Hagmar, L., Bjork, J., Sjodin, A., Bergman, A. \& Erfurth, E.M., Plasma levels of persistent organohalogens and hormone levels in adult male humans. Arch Environ Health, 56, pp. 138-143, 2001.

[22] Longnecker, M.P., Wolff, M.S., Gladen, B.C., Brock, J.W., Grandjean, P. \& Jacobson, J.L.e.a., Comparison of polychlorinated biphenyl levels across studies of human neurodevelopment. Environ Health Perspect, 111, pp. 65-70, 2003. 